\title{
Hemostasis in Acute and Chronic Liver Disease
}

\author{
Armando Tripodi, $\mathrm{PhD}^{1}$ \\ ${ }^{1}$ Angelo Bianchi Bonomi Hemophilia and Thrombosis Center, \\ Department of Clinical Sciences and Community Health, Università \\ degli Studi di Milano and IRCCS Cà Granda Maggiore Hospital \\ Foundation, Milano, Italy
}

Semin Liver Dis 2017;37:28-32.

\begin{abstract}
Address for correspondence A. Tripodi, PhD, Via Pace 9, 20122
Milano, Italy (e-mail: armando.tripodi@unimi.it).
\end{abstract}

\begin{abstract}
Acute and chronic liver diseases have long been considered prototypes of acquired hemorrhagic diseases. Over the last decade, evidence stemming from the laboratory bench and clinical practice has indicated that hemostasis abnormalities, until recently considered as the cause of bleeding in these conditions, are rebalanced to normal

Keywords

- hypocoagulability

- hypercoagulability

- thrombosis

- hemorrhage despite the abnormal results of the hemostasis tests such as prothrombin time and platelet counts. Consequently, the commonly used therapeutic approach-the infusion of plasma, platelets, or other prohemostatic agents-are not biologically plausible and should be reconsidered. In this article, the author reviews the evidence supporting the changing paradigm.
\end{abstract}

\section{Pathophysiology of Hemostasis}

Blood loss from injured vessels is prevented by hemostasis, a complex process that is tightly regulated by thrombin generation and fibrin deposition (also called coagulation), plateletvessel wall interaction (primary hemostasis), and fibrin dissolution (fibrinolysis). Perturbation of any of these processes may result in hemorrhage or thrombosis.

\section{Thrombin Generation and Fibrin Deposition (Coagulation)}

Thrombin generation and fibrin deposition (coagulation) is a tightly regulated process driven by procoagulant factors (from XII down to fibrinogen) that are counterbalanced by anticoagulant factors (e.g., antithrombin, protein C, protein S, and the tissue factor pathway inhibitor). The balance between pro- and anticoagulants prevents unwanted thrombin generation and fibrin deposition under physiological conditions. In contrast, perturbation of the balance that favors the procoagulants or the anticoagulants may lead to thrombosis or hemorrhage, respectively.

\section{Platelet-Vessel Wall Interaction (Primary Hemostasis)}

Platelets play a dual role in hemostasis.

First, they adhere to the subendothelium at the site of a vessel wall injury and form an aggregate of one another. Adhesion and aggregation are mediated by the adhesive multimeric protein von Willebrand factor (VWF), which in turn is regulated by ADAMTS13 (a disintegrin and metalloproteinase with a thrombospondin type 1 motif, member 13) that reduces its multimeric size and therefore platelet adhesiveness and aggregation. Defective platelet function (e. g., thrombocytopenia/thrombocytopathy) or VWF deficiency may result in hemorrhage, whereas increased platelet function (e.g., platelet hyperactivity, increased levels of VWF, or ADAMTS13 deficiency) may result in thrombosis.

Second, platelets support thrombin generation by assembling on their surface vitamin-K-dependent coagulation factors, a function known as platelet procoagulant activity. Defective platelet procoagulant activity may result in hemorrhage.

\section{Fibrin Dissolution (Fibrinolysis)}

Fibrinolysis is the system responsible for fibrin degradation that operates through two opposing drivers regulating the conversion of plasminogen (precursor) to plasmin (active enzyme) that ultimately digests fibrin. The profibrinolytic drivers include the tissue plasminogen activator (tPA), factor XIIa, and the urokinase plasminogen activator. The antifibrinolytic drivers include the thrombin-activatable fibrinolysis inhibitor (TAFI), the inhibitor to tPA (PAI), and the plasmin inhibitor. Under physiological conditions, the balance between the two opposing drivers prevents unwanted plasmin generation. Conversely, hypo- or hyperfibrinolysis may result in thrombosis or hemorrhage, respectively.
Copyright @ 2017 by Thieme Medical Publishers, Inc., 333 Seventh Avenue, New York, NY 10001, USA. Tel: +1(212) 584-4662.
DOI http://dx.doi.org/ 10.1055/s-0036-1597770. ISSN 0272-8087. 
Table 1 Hemostasis abnormalities observed in patients with liver disease

\begin{tabular}{|c|c|c|c|c|}
\hline \multirow[t]{2}{*}{ Mechanism } & \multicolumn{4}{|l|}{ Abnormality } \\
\hline & Acute liver disease & Status & Chronic liver disease & Status \\
\hline $\begin{array}{l}\text { Thrombin generation and fibrin } \\
\text { deposition (coagulation) }\end{array}$ & $\begin{array}{l}\text { Low procoagulants } \\
\text { High factor VIII } \\
\text { Low anticoagulants } \\
\text { High procoagulant } \\
\text { microparticles }\end{array}$ & Rebalanced & $\begin{array}{l}\text { Low procoagulants } \\
\text { High factor VIII } \\
\text { Low anticoagulants }\end{array}$ & Rebalanced \\
\hline $\begin{array}{l}\text { Platelet-vessel wall interaction } \\
\text { (primary hemostasis) }\end{array}$ & $\begin{array}{l}\text { Thrombocytopenia } \\
\text { High VWF } \\
\text { Low ADAMTS13 }\end{array}$ & Rebalanced & $\begin{array}{l}\text { Thrombocytopenia } \\
\text { High VWF } \\
\text { Low ADAMTS13 }\end{array}$ & Rebalanced \\
\hline Fibrin dissolution (fibrinolysis) & $\begin{array}{l}\text { Hypofibrinolysis } \\
\text { (established) }\end{array}$ & Not rebalanced & $\begin{array}{l}\text { Hyperfibrinolysis } \\
\text { (controversial) }\end{array}$ & Rebalanced? \\
\hline
\end{tabular}

Abbreviations: ADAMTS13, a disintegrin and metalloproteinase with a thrombospondin type 1 motif, member 13; VFW, von Willebrand factor.

Derangement of the above mechanisms has been reported to occur in liver disease. Here I will briefly review the hemostasis abnormalities in acute and chronic liver disease (-Table 1).

\section{Acute Liver Disease}

Acute liver disease (ALD) is a condition characterized by a sudden and severe deficiency of coagulation factors (both pro- and anticoagulants), ${ }^{1}$ thrombocytopenia, and systemic inflammation. ${ }^{2}$ Notably, the only coagulation factors that are not decreased (they are actually increased) are VWF and FVIII. They are probably increased because they are not synthesized by hepatocytes, but by endothelial cells.

\section{Thrombin Generation and Fibrin Deposition (Coagulation) in Acute Liver Disease}

Although hemorrhage in patients with ALD is not frequently observed, the condition has been considered for some time as a hemorrhagic disease because the prothrombin time (PT) in this setting is abnormally prolonged. Consequently, PT has been used as a prognostic index (PT prolongation is part of the definition of $\mathrm{ALD}^{3}$ ), but also as an index to guide transfusion (prophylaxis or rescue therapy) on the belief that correction of the PT would result in the clinical efficacy of fresh frozen plasma (FFP) or other prohemostatic agents. Recently, laboratory studies have shown that coagulation in patients with ALD is "rebalanced" despite PT prolongation. Thrombin-generation tests performed in platelet-poor plasma in the presence of exogenously added thrombomodulin showed that patients with ALD generate as much thrombin as healthy subjects. ${ }^{4,5}$ This was due to the commensurate deficiency of both pro- and anticoagulant factors, a typical feature of ALD. Hence, PT cannot be taken as a reliable index of hemorrhagic risk and cannot be used to guide transfusion using FFP or other prohemostatic agents. Prothrombin time, though sensitive to a deficiency of procoagulant factors (VII, X, II, and fibrinogen), is not sensitive to a deficiency of anticoagulant factors, which are concomitantly decreased in patients with liver disease. ${ }^{6}$ In fact, PT, when prolonged, can be used to diagnose patients with a congenital deficiency of procoagulants, but not to diagnose patients with a congenital deficiency of anticoagulants. The PT is within normal limits in patients with a congenital deficiency of naturally occurring anticoagulants-antithrombin, protein $\mathrm{C}$, or protein $\mathrm{S}-$ a condition where the PT should be shortened because these patients generate greater amounts of thrombin than normal subjects. Prothrombin time displays different sensitivity toward proand anticoagulants because upon triggering coagulation in vitro, procoagulants act quickly and limit thrombin generation and fibrin formation (i.e., the PT endpoint), whereas anticoagulants act at a much slower rate. ${ }^{6}$ For example, protein $\mathrm{C}$, one of the most powerful anticoagulants, circulates in plasma as a zymogen and is activated in vivo by thrombin in complex with its endothelial receptor thrombomodulin. ${ }^{7}$ Neither the reagent nor plasma used to measure the PT contains sufficient amounts of thrombomodulin. Therefore, protein $C$ that is markedly decreased in chronic liver disease (CLD) cannot be optimally activated within the relatively short time needed for PT execution (few seconds) and cannot exert its full anticoagulant activity as it occurs in vivo.

Platelet-Vessel Wall Interaction in Acute Liver Disease Although thrombocytopenia is not (in general) very severe in patients with ALD, it is observed in this setting. Although the mechanism has not yet been experimentally investigated, it can be surmised that the increased levels of VWF and reduced levels of ADAMTS13 are able to compensate for the mild thrombocytopenia observed in ALD. ${ }^{8}$ Hence, thrombocytopenia is not a crucial issue in patients with ALD.

\section{Fibrinolysis in Acute Liver Disease}

Recent evidence indicates that patients with ALD also have hypofibrinolysis, which might increase the risk of thrombosis. ${ }^{4}$ Thrombosis is in fact not an infrequent finding in patients with ALD. ${ }^{9}$ In addition to hypofibrinolysis, another factor that may trigger thrombosis is the reduced level of protein $C$ combined with an increased level of FVIII. Furthermore, the systemic inflammatory process that takes place in $\operatorname{ALD}^{10}$ may favor the tissue-factor-dependent activation of coagulation, 
which may also be triggered by procoagulant microparticles ${ }^{11}$ found in patients with ALD.

Recent observations suggest a changing paradigm of the hemostasis abnormalities associated with ALD. It is not, as previously believed, a hemorrhagic condition. It should rather be considered as a condition in which hemostasis is rebalanced. Therefore, the practice of infusing FFP or other prohemostatic agents to correct the abnormal hemostasis tests (PTor platelet counts) should be reconsidered, as it is not biologically plausible nor there is evidence that it is clinically effective. The infusion of FFP in particular might (paradoxically) "make a bad situation worse" because of fluid overload. The observation of a rebalanced hemostasis suggests also that patients with ALD are not "auto-anti-coagulated" as suggested by the prolonged PT and could therefore benefit from antithrombotic therapy (or prophylaxis) in the case of thrombosis.

\section{Chronic Liver Disease}

Chronic liver disease is characterized by a variable deficiency of coagulation factors (both pro- and anticoagulants). At variance with ALD, the deficiency in CLD is more likely due to synthetic defects rather than to consumption. As in ALD, the VWF and FVIII are increased in CLD. Thrombocytopenia is a distinct feature of CLD, and the platelet counts are in general smaller than those observed for ALD.

\section{Coagulation in Chronic Liver Disease}

\section{Hypocoagulability}

There is strong experimental evidence that coagulation (thrombin and fibrin formation) is rebalanced in CLD. Platelet-poor plasma from patients with CLD when tested in the presence of thrombomodulin generates normal amounts of thrombin despite the prolonged PT. ${ }^{12}$ In ALD-as in CLDcoagulation is rebalanced because of the commensurate deficiency of both pro- and anticoagulants. Therefore, the PT that is not representative of in vivo coagulation is not a reliable test of bleeding prediction or as a guide for transfusion therapy (as described previously). Furthermore, the observation of normal thrombin generation would question the common practice of treating patients with CLD with an infusion of FFP or other prohemostatic agents to correct the prolonged PT before an invasive procedure or as a rescue therapy in case of bleeding.

Controlled randomized trials have shown that recombinant activated factor VII (rFVIIa), a potent prohemostatic agent currently licensed for the treatment of patients with FVIII inhibitors, poorly stops or prevents bleeding in patients (1) who have bleeding esophageal varices, ${ }^{13-15}$ or (2) were undergoing hepatectomy. ${ }^{16,17}$ Although there are no controlled randomized trials on the efficacy/safety of FFP in patients with CLD, a recent in vitro study investigated plasmas from patients with CLD to which a normal pooled plasma was added at a proportion similar to that obtained in vivo when $15 \mathrm{~mL} / \mathrm{kg}$ of FFP is infused. Pre- and postaddition plasmas were analyzed for PT and thrombin generation. Although the PT was somewhat shortened (though not completely normalized) at postinfusion, thrombin generation remained unchanged. ${ }^{18}$ These in vitro experiments cast doubt on the efficacy of FFP in this setting. Furthermore, the indiscriminate infusion of FFP in these patients could increase portal hypertension, thus (paradoxically) increasing the risk of bleeding.

\section{Hypercoagulability}

When thrombin generation was measured with and without the addition of thrombomodulin in the plasma of patients with CLD, the endogenous thrombin potential (ETP; the main parameter of a thrombin generation assay) was expressed as a ratio-with or without thrombomodulin. This ratio was significantly higher in patients than in the controls. ${ }^{19}$ The ETP ratio is considered an index of plasma hypercoagulability (by definition: the higher the ETP ratio, the greater the hypercoagulability). ${ }^{20}$ The ETP ratio in patients with CLD was directly correlated with the increased levels of FVIII (the most potent driver of thrombin generation) and inversely correlated with the decreased levels of protein $C$ (the most important driver of thrombin inhibition). ${ }^{19}$ These observations suggest that patients with CLD possess a procoagulant imbalance stemming from an in vitro resistance to thrombomodulin that can be explained by the increased levels of FVIII and decreased levels of protein $C$ that are typical features of patients with CLD. Whether the in vitro resistance to thrombomodulin promotes the increased risk of venous thromboembolism that has been observed in patients with $\operatorname{CLD}^{21,22}$ is still unknown. Preliminary retrospective observations showed that patients with CLD who had high ETP ratios were at higher risk to develop de novo episodes of portal vein thrombosis. ${ }^{23}$ The occurrence of hypercoagulability in patients with CLD may have practical implications outside thrombosis as well. Thrombin has been identified as a possible mediator of the progression of liver fibrosis in patients with CLD that might be responsible for parenchymal extinction. ${ }^{24}$

These study results cast serious doubts on the validity of infusion of FFP or other prohemostatic agents such as rFVIIa or prothrombin complex concentrates in patients with CLD. Furthermore, they also show that patients with CLD are not auto-anti-coagulated as has been believed; therefore, they should be treated with anticoagulants to prevent or treat thrombosis. A recent clinical trial of patients awaiting liver transplantation showed that antithrombotic prophylaxis with low-molecular-weight heparin was effective in preventing portal vein thrombosis without significant bleeding events. ${ }^{25}$

\section{Platelet-Vessel Wall Interaction in Chronic Liver Disease}

There is strong experimental evidence that in addition to coagulation, primary hemostasis is rebalanced in CLD. Lisman et $\mathrm{al}^{26}$ showed that platelets from patients with CLD adhere normally to collagen in an in vitro system operating under flow conditions. The authors concluded that elevated levels of VWF observed in these patients compensated for the relative thrombocytopenia observed in this setting.

As mentioned, platelets support thrombin generation by exposing on their surface negatively charged phospholipids 
that bind vitamin-K-dependent coagulation factors, thus speeding up thrombin generation. Tripodi et $\mathrm{al}^{27}$ showed that thrombin generation in platelet-rich plasma from patients with CLD was similar to that of healthy subjects, provided that platelet counts were adjusted to a standard count of $100 \times 10^{9} / \mathrm{L}^{27}$ The platelet count that generated thrombin amounts equivalent to the lower limit of the normal range was $56 \times 10^{9} / \mathrm{L}^{27}$ The data suggest that platelets from patients with CLD are qualitatively able to support normal thrombin generation if patients are not severely thrombocytopenic. In line with these observations, the American Association for the Study of the Liver in a recent position paper recommends correcting platelet counts if they are lower than $50 \times 10^{9} /$ L before liver biopsy. ${ }^{28}$ A recent study investigated the effect of a single infusion of an adult platelet unit in patients with CLD before undergoing invasive procedures when their baseline platelet counts were $<50 \times 10^{9} / \mathrm{L}$. Postinfusion platelet counts were marginally increased, but thrombin generation in platelet-rich plasma or thromboelastography in whole blood remained unchanged. ${ }^{29}$ These observations suggest that if platelets are to be increased to an extent sufficient to modify functional platelet assays, multiple transfusions are required, or alternatively, platelet counts should be increased by nontransfusional approaches with the administration of agonists of the thrombopoietin receptor such as eltrombopag. This drug has been successfully used to increase platelet numbers in patients with immune thrombocytopenia ${ }^{30}$ and in patients with hepatitis $C$ virus infection before antiviral therapy. ${ }^{31}$ Recently, eltrombopag was administered to patients with CLD, who were thrombocytopenic and required invasive procedures. ${ }^{32}$ The drug was effective in increasing platelet numbers, but the study was terminated because of an excessive rate of portal vein thromboses in patients treated with the drug. ${ }^{32}$

A decision should be made about a transfusional or nontransfusional approach in CLD only after careful consideration of the benefit/risk ratio in individual patients. ${ }^{33}$

\section{Fibrinolysis in Chronic Liver Disease}

Little information is available on fibrinolysis in patients with CLD. Early studies reported that hyperfibrinolysis was associated with cirrhosis. ${ }^{34}$ However, in those studies fibrinolysis was assessed by measuring pro- or antifibrinolytic parameters that (by definition) cannot account for the balance between the pro- and antifibrinolytic drivers operating in vivo. Recent studies have investigated fibrinolysis in patients with CLD by global assays. ${ }^{35-37}$ The results have been inconsistent: Two studies concluded that patients with CLD possess hyperfibrinolysis, mostly due to the considerable reduction of $\mathrm{TAFI}^{36,37}$; one study concluded that fibrinolysis is substantially rebalanced because of the parallel reduction of both pro- and antifibrinolytic drivers. ${ }^{35}$ It is therefore still unclear whether hyperfibrinolysis exists in patients with CLD and whether it is responsible for the bleeding events occasionally observed in these patients. On the other hand, controlled randomized clinical trials of antifibrinolytic drugs (e.g., tranexamic acid or epsilon aminocaproic acid) for controlling bleeding in this setting have never been performed. ${ }^{38}$

\section{Conclusions}

Experimental observations and clinical practice show that hemostasis in ALD and CLD is probably rebalanced by the commensurate reduction of both pro- and anticoagulants and by the increased levels of VWF and FVIII. Consequently, both conditions that were until recently considered as the prototypes of acquired hemorrhagic diseases should be reconsidered with important practical implications. For instance, the bleeding events observed in patients with CLD are not due to hemostasis derangement, but probably to other conditions that underlie CLD such as portal hypertension, recurrent bacterial infections, endothelial dysfunction, or hepatorenal syndrome. These conditions need to be treated and new therapeutic strategies to control bleeding in patients with liver disease developed. In addition, although hemostasis is substantially rebalanced, it is not as stable as in healthy individuals; therefore, it may cause hemorrhage or thrombosis depending on the circumstantial risk factors. The above observations make liver disease shift from purely hemorrhagic to a mixed and thrombosis-liable disease.

\section{Abbreviations}

ADAMTS13 a disintegrin and metalloproteinase with a thrombospondin type 1 motif, member 13

ALD acute liver disease

CLD chronic liver disease

ETP endogenous thrombin potential

FFP fresh frozen plasma

FVIII factor VIII

PAI inhibitor to tPA

PT prothrombin time

rFVIIa recombinant activated factor VII

TAFI thrombin-activatable fibrinolysis inhibitor

tPA tissue plasminogen activator

VWF von Willebrand factor.

\section{References}

1 Kerr R, Newsome P, Germain L, et al. Effects of acute liver injury on blood coagulation. J Thromb Haemost 2003;1(4):754-759

2 Stravitz RT, Ellerbe C, Durkalski V, Reuben A, Lisman T, Lee WM; Acute Liver Failure Study Group. Thrombocytopenia is associated with multi-organ system failure in patients with acute liver failure. Clin Gastroenterol Hepatol 2016;14(4):613-620.e4

3 Wlodzimirow KA, Eslami S, Abu-Hanna A, Nieuwoudt M, Chamuleau RA. Systematic review: acute liver failure - one disease, more than 40 definitions. Aliment Pharmacol Ther 2012;35(11):1245-1256

4 Lisman T, Bakhtiari K, Adelmeijer J, Meijers JC, Porte RJ, Stravitz RT. Intact thrombin generation and decreased fibrinolytic capacity in patients with acute liver injury or acute liver failure. J Thromb Haemost 2012;10(7):1312-1319

5 Habib M, Roberts LN, Patel RK, Wendon J, Bernal W, Arya R. Evidence of rebalanced coagulation in acute liver injury and acute liver failure as measured by thrombin generation. Liver Int 2014; 34(5):672-678

6 Tripodi A, Chantarangkul V, Mannucci PM. Acquired coagulation disorders: revisited using global coagulation/anticoagulation testing. Br J Haematol 2009;147(1):77-82 
7 Dahlbäck B, Villoutreix BO. Regulation of blood coagulation by the protein $C$ anticoagulant pathway: novel insights into structurefunction relationships and molecular recognition. Arterioscler Thromb Vasc Biol 2005;25(7):1311-1320

8 Hugenholtz GC, Adelmeijer J, Meijers JC, Porte RJ, Stravitz RT, Lisman T. An unbalance between von Willebrand factor and ADAMTS13 in acute liver failure: implications for hemostasis and clinical outcome. Hepatology 2013;58(2):752-761

9 Stravitz RT, Lisman T, Luketic VA, et al. Minimal effects of acute liver injury/acute liver failure on hemostasis as assessed by thromboelastography. J Hepatol 2012;56(1):129-136

10 Rolando N, Wade J, Davalos M, Wendon J, Philpott-Howard J, Williams R. The systemic inflammatory response syndrome in acute liver failure. Hepatology 2000;32(4 Pt 1):734-739

11 Stravitz RT, Bowling R, Bradford RL, et al. Role of procoagulant microparticles in mediating complications and outcome of acute liver injury/acute liver failure. Hepatology 2013;58(1):304-313

12 Tripodi A, Salerno F, Chantarangkul V, et al. Evidence of normal thrombin generation in cirrhosis despite abnormal conventional coagulation tests. Hepatology 2005;41(3):553-558

13 Bosch J, Thabut D, Bendtsen F, et al; European Study Group on rFVIIa in UGI Haemorrhage. Recombinant factor VIIa for upper gastrointestinal bleeding in patients with cirrhosis: a randomized, double-blind trial. Gastroenterology 2004;127(4):1123-1130

14 Bosch J, Thabut D, Albillos A, et al; International Study Group on rFVIIa in UGI Hemorrhage. Recombinant factor VIIa for variceal bleeding in patients with advanced cirrhosis: a randomized, controlled trial. Hepatology 2008;47(5):1604-1614

15 Bendtsen F, D'Amico G, Rusch E, et al. Effect of recombinant Factor VIIa on outcome of acute variceal bleeding: an individual patient based meta-analysis of two controlled trials. J Hepatol 2014;61(2): 252-259

16 Lodge JP, Jonas S, Jones RM, et al; rFVIIa OLT Study Group. Efficacy and safety of repeated perioperative doses of recombinant factor VIla in liver transplantation. Liver Transpl 2005;11(8):973-979

17 Planinsic RM, van der Meer J, Testa G, et al. Safety and efficacy of a single bolus administration of recombinant factor VIIa in liver transplantation due to chronic liver disease. Liver Transpl 2005; 11(8):895-900

18 Tripodi A, Chantarangkul V, Primignani M, et al. Thrombin generation in plasma from patients with cirrhosis supplemented with normal plasma: considerations on the efficacy of treatment with fresh-frozen plasma. Intern Emerg Med 2012;7(2):139-144

19 Tripodi A, Primignani M, Chantarangkul V, et al. An imbalance of pro- vs anti-coagulation factors in plasma from patients with cirrhosis. Gastroenterology 2009;137(6):2105-2111

20 Dargaud Y, Trzeciak MC, Bordet JC, Ninet J, Negrier C. Use of calibrated automated thrombinography $+/$ - thrombomodulin to recognise the prothrombotic phenotype. Thromb Haemost 2006; 96(5):562-567

21 Northup PG, McMahon MM, Ruhl AP, et al. Coagulopathy does not fully protect hospitalized cirrhosis patients from peripheral venous thromboembolism. Am J Gastroenterol 2006;101(7): 1524-1528, quiz 1680

22 Søgaard KK, Horváth-Puhó E, Grønbaek H, Jepsen P, Vilstrup H, Sørensen HT. Risk of venous thromboembolism in patients with liver disease: a nationwide population-based case-control study. Am J Gastroenterol 2009;104(1):96-101

23 La Mura V, Tripodi A, Tosetti G, et al. Resistance to thrombomodulin is associated with de novo portal vein thrombosis and low survival in patients with cirrhosis. Liver Int 2016;36(9): $1322-1330$

24 Tripodi A, Anstee QM, Sogaard KK, Primignani M, Valla DC. Hypercoagulability in cirrhosis: causes and consequences. J Thromb Haemost 2011;9(9):1713-1723

25 Villa E, Cammà C, Marietta M, et al. Enoxaparin prevents portal vein thrombosis and liver decompensation in patients with advanced cirrhosis. Gastroenterology 2012;143(5):1253-60. e1, 4

26 Lisman T, Bongers TN, Adelmeijer J, et al. Elevated levels of von Willebrand Factor in cirrhosis support platelet adhesion despite reduced functional capacity. Hepatology 2006;44(1):53-61

27 Tripodi A, Primignani M, Chantarangkul V, et al. Thrombin generation in patients with cirrhosis: the role of platelets. Hepatology 2006;44(2):440-445

28 Rockey DC, Caldwell SH, Goodman ZD, Nelson RC, Smith AD; American Association for the Study of Liver Diseases. Liver biopsy. Hepatology 2009;49(3):1017-1044

29 Tripodi A, Primignani M, Chantarangkul V, et al. Global hemostasis tests in patients with cirrhosis before and after prophylactic platelet transfusion. Liver Int 2013;33(3):362-367

30 Gómez-Almaguer D, Herrera-Rojas MA, Jaime-Pérez JC, et al. Eltrombopag and high-dose dexamethasone as frontline treatment of newly diagnosed immune thrombocytopenia in adults. Blood 2014;123(25):3906-3908

31 Afdhal NH, Dusheiko GM, Giannini EG, et al. Eltrombopag increases platelet numbers in thrombocytopenic patients with HCV infection and cirrhosis, allowing for effective antiviral therapy. Gastroenterology 2014;146(2):442-52.e1

32 Afdhal NH, Giannini EG, Tayyab G, et al; ELEVATE Study Group. Eltrombopag before procedures in patients with cirrhosis and thrombocytopenia. N Engl J Med 2012;367(8):716-724

33 Tripodi A, Primignani M. Nontransfusional approach to increased platelet count in patients with cirrhosis and thrombocytopenia. Hepatology 2013;58(3):1177-1180

34 Violi F, Ferro D, Basili S, et al. Hyperfibrinolysis increases the risk of gastrointestinal hemorrhage in patients with advanced cirrhosis. Hepatology 1992;15(4):672-676

35 Lisman T, Leebeek FW, Mosnier LO, et al. Thrombin-activatable fibrinolysis inhibitor deficiency in cirrhosis is not associated with increased plasma fibrinolysis. Gastroenterology 2001;121(1): 131-139

36 Colucci M, Binetti BM, Branca MG, et al. Deficiency of thrombin activatable fibrinolysis inhibitor in cirrhosis is associated with increased plasma fibrinolysis. Hepatology 2003;38(1):230-237

37 Rijken DC, Kock EL, Guimarães AH, et al. Evidence for an enhanced fibrinolytic capacity in cirrhosis as measured with two different global fibrinolysis tests. J Thromb Haemost 2012;10(10): 2116-2122

38 Martí-Carvajal AJ, Solà I. Antifibrinolytic amino acids for upper gastrointestinal bleeding in people with acute or chronic liver disease. Cochrane Database Syst Rev 2015;6(6):CD006007 